\title{
Efficacy of phosphatidic acid ingestion on lean body mass, muscle thickness and strength gains in resistance-trained men
}

\author{
Jay R Hoffman ${ }^{1 *}$, Jeffrey R Stout ${ }^{1}$, David R Williams ${ }^{1}$, Adam J Wells ${ }^{1}$, Maren S Fragala ${ }^{1}$, Gerald T Mangine ${ }^{1}$, \\ Adam M Gonzalez', Nadia S Emerson' ${ }^{1}$, William P McCormack', Tyler C Scanlon' ${ }^{1}$ Martin Purpura² and Ralf Jäger ${ }^{2}$
}

\begin{abstract}
Background: Phosphatidic acid (PA) has been reported to activate the mammalian target of rapamycin (mTOR) signaling pathway and is thought to enhance the anabolic effects of resistance training. The purpose of this pilot study was to examine if oral phosphatidic acid administration can enhance strength, muscle thickness and lean tissue accruement during an 8-week resistance training program.

Methods: Sixteen resistance-trained men were randomly assigned to a group that either consumed $750 \mathrm{mg}$ of PA $(n=7,23.1 \pm 4.4 y ; 176.7 \pm 6.7 \mathrm{~cm} ; 86.5 \pm 21.2 \mathrm{~kg})$ or a placebo $(P L, n=9,22.5 \pm 2.0 \mathrm{y} ; 179.8 \pm 5.4 \mathrm{~cm} ; 89.4 \pm 13.6 \mathrm{~kg})$ group. During each testing session subjects were assessed for strength (one repetition maximum [1-RM] bench press and squat) and body composition. Muscle thickness and pennation angle were also measured in the vastus lateralis of the subject's dominant leg.

Results: Subjects ingesting PA demonstrated a $12.7 \%$ increase in squat strength and a $2.6 \%$ increase in LBM, while subjects consuming PL showed a 9.3\% improvement in squat strength and a $0.1 \%$ change in LBM. Although parametric analysis was unable to demonstrate significant differences, magnitude based inferences indicated that the $\Delta$ change in 1-RM squat showed a likely benefit from PA on increasing lower body strength and a very likely benefit for increasing lean body mass (LBM).

Conclusions: Results of this study suggest that a combination of a daily $750 \mathrm{mg}$ PA ingestion, combined with a 4-day per week resistance training program for 8-weeks appears to have a likely benefit on strength improvement, and a very likely benefit on lean tissue accruement in young, resistance trained individuals.
\end{abstract}

Keywords: Nutritional supplement, Muscle architecture, Ergogenic aid, Phospholipid, Resistance training

\section{Introduction}

Phospholipids are a major structural component of all biological membrane systems [1,2]. Phosphatidic acid (PA) or 1,2-diacyl-sn-glycero-3-phosphate is a phospholipid that makes up a small percentage of the total phospholipid pool [3-5]. It not only is a constituent of all cell membranes, it also acts as an intermediate in the biosynthesis of triacylglycerols and other phospholipids. It is also suggested to act as an intracellular lipid second messenger that regulates signaling proteins, including

\footnotetext{
* Correspondence: Jay.Hoffman@ucf.edu

${ }^{1}$ Human Performance Laboratory, University of Central Florida, Orlando 32186, FL, USA

Full list of author information is available at the end of the article
}

several kinases and phosphatases [3,6,7]. One of the signaling proteins that PA has been suggested to stimulate is mammalian target of rapamycin (mTOR) [8,9], a serine threonine kinase that integrates metabolic signals from various factors including protein metabolism and cytoskeleton organization that controls cell growth [10]. Both nutritional and mechanical stimuli have been implicated in stimulating this pathway. These different stimuli appear to act at different substrate levels either upstream or downstream from mTOR. Hornberger and colleagues have suggested that the mechanical activation from external loads (as one may see from a resistance exercise session) may be enhanced with the presence of PA [11]. It has been shown that exogenous supplied PA

\section{() Bïomed Central}


can stimulate the mTOR pathway via its activation of the substrate S6 kinase [4,7]. Interestingly, the binding of PA to S6 kinase may occur independently of mTOR [12], suggesting that PA may augment the signaling response when mTOR is activated by exercise. These data provide an interesting hypothesis that the ingestion of $\mathrm{PA}$, in combination with a resistance training program, may stimulate potentially greater gains in muscle strength and growth than resistance training alone.

The ability to augment muscle strength and size has important implications for various population groups. Specifically, the ability for a dietary supplement to enhance muscle strength and increase lean mass would be of consequence for competitive athletes who are focused on maximizing strength and size gains, and older adults who are battling the effects of aging and sarcopenia. Presently, there does not appear to be any study available that has examined effect of PA supplementation on strength and lean tissue adaptation. Therefore, it is the purpose of this pilot study to examine if PA ingestion can enhance strength, muscle thickness and lean tissue accruement during an 8-week resistance training program more so than training only.

\section{Methods}

\section{Subjects}

Twenty resistance-trained men (at least 1 year of training experience) volunteered to participate in this randomized, double-blind, placebo-controlled, repeated measures study. None of the subjects were competitive strength/power athletes, but all subjects were currently engaged in recreational weight lifting that included using the squat and bench press exercises. Following an explanation of all procedures, risks and benefits, each subject gave his informed written consent prior to participating in this study. The University Institutional Review Board approved the research protocol. Subjects were asked to not use any anabolic dietary supplements or drugs know to increase muscle and/or performance.
Screening for dietary supplements or drugs was accomplished by a health questionnaire filled out during subject recruitment.

Subjects were randomly assigned to one of two treatment groups, $750 \mathrm{mg}$ phosphatidic acid (PA; $23.1 \pm 4.4 \mathrm{y}$; $176.7 \pm 6.7 \mathrm{~cm} ; 86.5 \pm 21.2 \mathrm{~kg})$ or $750 \mathrm{mg}$ rice flour, which served as placebo (PL; $22.5 \pm 2.0 \mathrm{y} ; 179.8 \pm 5.4 \mathrm{~cm}$; $89.4 \pm 13.6 \mathrm{~kg}$ ). Four subjects were dropped from the study. One of the subjects was injured during a recreational activity, another subject dropped out due to a family crisis, and the other two subjects were removed due to a lack of compliance. A total of 7 subjects remained in the PA group and 9 subjects in the PL group. The PA supplement (Mediator ${ }^{\mathrm{TM}}$ ) was obtained from Chemi Nutra (White Bear Lake, MN). Both the PA and PL were in capsule form and were similar in appearance. Subjects were provided a weekly capsule allotment and returned the bottle at the end of the week to receive their next week's supply. Subjects were required to consume five capsules of either the treatment once per day ad libitum. Timing of capsule ingestion was not controlled. Each capsule contained $150 \mathrm{mg}$ of PA or PL. To standardize post-workout protein ingestion, all subjects were provided a 36-g amino acid and collagen protein blend (see Table 1 for content) mixed in a $500 \mathrm{ml}$ commercial sports drink. This drink was consumed within 30 minutes post-exercise.

All groups performed the same 4-day per week, split routine resistance training program for 8-weeks (see Table 2). The subjects were required to exercise with $70 \%$ of their 1-repetition maximum (1-RM) for all exercises. The load for the assistance exercises was selfdetermined by the subject, but they were required to use a load that allowed them to perform a 10-12 RM. A 90$s$ rest period was required between each set, for all exercises. Subjects trained at their local gym off campus without investigator supervision. However, all subjects maintained a daily training log and turned it in at the end of each week. Feedback to subjects on training logs

Table 1 Post-workout amino acid and collagen protein blend ingredients

\begin{tabular}{lcl}
\hline Amino acid & g AA/100 g of product & Amino acid \\
\hline Alanine & 7.6 & Leucine \\
\hline Arginine & 7.8 & Lysine \\
\hline Aspartic acid & 5.1 & Methionine \\
\hline Cystine & 0.0 & Phenylalanine \\
\hline Glutamic acid & 10.5 & Proline \\
\hline Glycine & 18.2 & Serine \\
\hline Histidine & 1.2 & Threonine \\
\hline Hydroxylysine & 0.5 & Tryptophan \\
\hline Hydroxylproline & 10.8 & Tyrosine \\
\hline Isoleucine & 1.4 & Valine \\
\hline
\end{tabular}


Table 2 Eight-week resistance training protocol

\begin{tabular}{lclr}
\hline Monday/Thursday & & Tuesday/Friday & Sets/Reps (RM) \\
\cline { 2 - 3 } Exercise & Sets/Reps (RM) & Exercise & $1,4 \times 10-12$ \\
\hline Bench Press* & $1,4 \times 10-12$ & Squats* & $3 \times 10-12$ \\
\hline Incline DB Press & $3 \times 10-12$ & Lunge/Front squat & $3 \times 10-12$ \\
\hline Seated Shoulder Press* & $1,4 \times 10-12$ & Leg Curl & $3 \times 10-12$ \\
\hline Upright rows & $3 \times 10-12$ & Knee Extension & $3 \times 10-12$ \\
\hline Lateral raises & $3 \times 10-12$ & Calf Raises & $4 \times 10-12$ \\
\hline Shrugs & $3 \times 10-12$ & Lat Pulldown & $4 \times 10-12$ \\
\hline Triceps pushdown & $3 \times 10-12$ & Seated Row & $3 \times 10-12$ \\
\hline Triceps extension & $3 \times 10-12$ & EZ Bar Curl & $3 \times 10-12$ \\
\hline Situps & $3 \times 25$ & Dumbbell Curls & $3 \times 25$ \\
\hline
\end{tabular}

was provided by certified study personnel. This insured appropriate changes to loading during the 8-week program.

\section{Testing protocol}

Subjects reported to the Human Performance Laboratory on two separate occasions. The first testing session occurred prior to the onset of supplementation, while the second testing session occurred at the conclusion of the 8-week supplementation program. All testing sessions occurred at the same time of day, and subjects were requested to maintain a similar daily routine on testing dates. Body composition and ultrasonography assessments were performed prior to all strength measures.

\section{Body composition}

Body composition was determined using whole bodydual energy $\mathrm{x}$-ray absorptiometry (DEXA) scans (Prodigy ${ }^{\mathrm{TM}}$; Lunar Corporation, Madison, WI). Total body estimates of percent fat, fat and non-bone lean tissue was determined using company's recommended procedures and supplied algorithms. Quality assurance was assessed by daily calibrations and was performed prior to all scans using a calibration block provided by the manufacturer.

\section{Strength measures}

During each testing session, subjects performed a 1-RM strength test for the squat and bench press exercises. The 1 RM tests were conducted as previously described by Hoffman [13]. Each subject performed a warm-up set using a resistance that was approximately $40-60 \%$ of his perceived maximum, and then performed 3-4 subsequent attempts to determine the 1-RM. A $3-5$ minute rest period was provided between each lift. No bouncing was permitted for the bench press exercise, as this would have artificially increased strength values. Bench press testing was performed in the standard supine position: the subject lowered an Olympic weight lifting bar to mid-chest level and then pressed the weight until his elbows were fully extended. The squat exercise required the subject to rest an Olympic weightlifting bar across the trapezius at a self-chosen location. The squat was performed to the parallel position (that was closely monitored by certified staff), which was achieved when the greater trochanter of the femur was lowered to the same level as the knee. The subject then lifted the weight until his knees were extended. Previous studies have demonstrated good test-retest reliabilities $(R>0.97)$ for these strength measures $[14,15]$.

\section{Ultrasonography measurements}

Skeletal muscle architecture was assessed on the subject's self-reported dominant leg using B-mode ultrasound imaging (General Electric LOGIQ P5) with a $12-\mathrm{MHz}$ linear probe. A water-soluble gel was applied to the probe. Images were obtained, as previously described [16], by the same technician for all tests using longitudinal probe positioning. Equal contact pressure was maintained during each measure. Vastus lateralis (VS) fascicle thickness and pennation angle were measured at $50 \%$ of femur length over the midbelly of the muscle with the subjects lying in a supine position. Pennation angle was determined by the angle between the deep aponeurosis and the fascicles [17]. Muscle thickness was determined as the distance between the subcutaneous adipose tissue and intermuscular interface. All ultrasonography measures were performed prior to the strength tests. The intraclass correlation coefficients \pm SEM for muscle thickness and pennation angle were $0.99 \pm .03$ and $0.95 \pm 0.91$, respectively.

\section{Dietary recall}

Three-day dietary records were completed during the week prior to the onset of the study. Subjects were 
instructed to record as accurately as possible everything they consumed during the day, including between meal and late evening snacks. FoodWorks Dietary Analysis software version 13 (The Nutrition Company, Long Valley, NJ) was used to analyze dietary recalls. Subjects were required to maintain their normal diet throughout the study.

\section{Statistical analysis}

Seven separate two-way mixed factorial Analysis of Variance (time [PRE, POST] $\times$ group [PA and PL]) were used to analyze the body mass (BM), body fat, lean body mass, vastus lateralis thickness and pennation angle, 1-RM bench press and squat data. In the event of a significant F- ratio, Tukey post-hoc tests were used for pairwise comparisons. For effect size, the partial eta squared statistic was reported and according to Green et al. [18], $0.01,0.06$, and 0.14 represents small, medium, and large effect sizes, respectively. An alpha level was set at $\mathrm{p} \leq 0.05$, and all analyses were performed using PASW version 18.0 (SPSS, Inc., Chicago, IL).

Recent investigations in sport science have suggested that the use of null-hypothesis testing may be inadequate for assessing clinical or practical significance $[19,20]$. An analysis that infers the magnitude of differences in means may provide a more qualitative interpretation of results. To make inferences on true effects of PA on strength and body composition, a published spreadsheet using the unequal variances t-statistic was used [19]. The effect of PA was calculated as the change score by calculating the difference between the post- and presupplementation scores for the PA and PL groups. The precision of the magnitude inference was set at $90 \%$ confidence limits, using the p-value corresponding to the $t$ statistic. The published spreadsheet calculated inferences whether the true population effect was substantially beneficial, harmful, or trivial based on the range of the confidence interval relative to the value for the smallest clinical worthwhile effect. An effect was reported to be unclear if the confidence interval overlapped the thresholds for positive and negative substantiveness $(>5 \%$ chance that the value was both substantially positive and negative). Or, the chance that the value was positive or negative was evaluated by: $<1 \%$, almost certainly not; 1 $5 \%$, very unlikely; $5-25 \%$, unlikely; $25-75 \%$, possible; $75-$ 95\%, likely; $95-99 \%$ very likely; and $>99 \%$ almost certain. Results were interpreted using magnitude-based statistics, using Cohen's thresholds ( $<0.1$, trivial; 0.1-0.3, small; 0.3-0.5, moderate; $>0.5$ large) [20].

\section{Results}

No significant differences were seen in caloric intake between PA $(3153 \pm 778 \mathrm{kcal})$ and PL $(3387 \pm 1168 \mathrm{kcal})$. In addition, no significant differences were seen in carbohydrate $(285 \pm 74 \quad$ g vs. $342 \pm 94$ g), protein $(227 \pm 68 \mathrm{~g}$ vs. $192 \pm 59 \mathrm{~g})$ and fat $(125 \pm 47 \mathrm{~g}$ vs. $136 \pm 77 \mathrm{~g}$ ) intakes between PA and PL, respectively. PA and PL were very well tolerated and no adverse events have been reported.

Pre to post changes in strength, muscle architecture and body composition are depicted in Table 3. Significant main effects (Pre vs. Post) were seen in both 1-RM bench press $(p=0.006)$ and 1-RM squat $(p=0.001)$ for both groups combined. However, no significant interactions were observed. A significant main effect was also observed for vastus lateralis thickness $(p=0.001)$, but not for pennation angle $(\mathrm{p}=0.156)$. No significant interactions were noted in either variable. No change in body mass ( $p=0.253$ ) was seen following eight weeks of training in either group, but a significant main effect was noted in the change in lean body mass $(\mathrm{p}=0.045)$. A trend $(\mathrm{p}=0.065)$ towards a significant interaction was observed for in lean body mass. The post hoc power analyses (Table 4) ndicated that values ranged from 0.05 to 0.46 for all group X time interactions and 0.05 to 0.97 for main effects for time.

Magnitude based inferences on changes in performance and anthropometric measures are described in Table 5 . The $\Delta$ change in 1-RM squat show a likely benefit from PA on increasing lower body strength. Subjects ingesting PA demonstrated a $12.7 \%$ in squat strength, while subjects consuming PL showed a 9.3\% improvement (See Figure 1). Improvements in 1-RM bench press were $5.1 \%$ and $3.3 \%$ in PA and PL, respectively. Magnitude based inferences were unclear regarding any benefit in upper body strength improvements in these subjects consuming the PA. Differences in the

Table 3 Strength, muscle architecture and body composition changes

\begin{tabular}{llll}
\hline Variable & Group & PRE & POST \\
\hline 1-RM Bench Press (kg) & $\mathrm{PA}$ & $122.1 \pm 21.6$ & $128.3 \pm 21.6$ \\
\cline { 2 - 4 } & $\mathrm{PL}$ & $115.2 \pm 29.6$ & $119.0 \pm 28.6$ \\
\hline 1-RM Squat (kg) & $\mathrm{PA}$ & $134.5 \pm 44.1$ & $151.6 \pm 41.1$ \\
\cline { 2 - 4 } & $\mathrm{PL}$ & $138.9 \pm 32.9$ & $151.8 \pm 33.9$ \\
\hline Vastus Lateralis Thickness (cm) & $\mathrm{PA}$ & $2.10 \pm 0.43$ & $2.41 \pm 0.27$ \\
\cline { 2 - 4 } & $\mathrm{PL}$ & $1.94 \pm 0.41$ & $2.24 \pm 0.54$ \\
\hline Vastus Lateralis Pennation angle $\left(^{\circ}\right)$ & $\mathrm{PA}$ & $16.49 \pm 2.95$ & $18.34 \pm 3.09$ \\
\cline { 2 - 4 } & $\mathrm{PL}$ & $15.6 \pm 3.28$ & $16.7 \pm 4.21$ \\
\hline Body Mass (kg) & $\mathrm{PA}$ & $86.5 \pm 21.2$ & $88.0 \pm 18.9$ \\
\cline { 2 - 4 } & $\mathrm{PL}$ & $89.4 \pm 13.6$ & $89.5 \pm 13.4$ \\
\hline Body Fat (kg) & $\mathrm{PA}$ & $15.8 \pm 15.4$ & $15.9 \pm 13.6$ \\
\cline { 2 - 4 } & $\mathrm{PL}$ & $17.5 \pm 9.4$ & $17.5 \pm 9.3$ \\
\hline Lean Body Mass (kg) & $\mathrm{PA}$ & $66.2 \pm 4.5$ & $67.9 \pm 5.6$ \\
\cline { 2 - 4 } & $\mathrm{PL}$ & $68.4 \pm 11.2$ & $68.5 \pm 11.2$ \\
\hline
\end{tabular}


Table 4 Statistical estimates for the dependent variables in this study

\begin{tabular}{|c|c|c|c|c|}
\hline Variable & $\mathbf{p}$ & $\mathbf{F}$ & $\begin{array}{l}\text { Effect } \\
\text { size }\end{array}$ & $\begin{array}{l}\text { Observed } \\
\text { power }\end{array}$ \\
\hline \multicolumn{5}{|l|}{ 1-RM Bench Press (Kg) } \\
\hline Group $x$ time interaction & 0.43 & 0.60 & 0.04 & 0.11 \\
\hline Group Time Effect & $0.006^{*}$ & 0.4 & 0.43 & 0.85 \\
\hline \multicolumn{5}{|l|}{ 1-RM Squat (Kg) } \\
\hline Group $x$ time interaction & 0.19 & 1.92 & 0.12 & 0.25 \\
\hline Group Time Effect & $0.00^{*}$ & 93.1 & 0.87 & 1.0 \\
\hline \multicolumn{5}{|c|}{ Vastus Lateralis Thickness (CM) } \\
\hline Group $x$ time interaction & 0.96 & 0.002 & 0.00 & 0.05 \\
\hline Group Time Effect & $0.001^{*}$ & 17.1 & 0.55 & 0.97 \\
\hline \multicolumn{5}{|c|}{ Vastus Lateralis Pennation angle $\left(^{\circ}\right)$} \\
\hline Group $x$ time interaction & 0.69 & 0.16 & 0.01 & 0.07 \\
\hline Group Time Effect & 0.16 & 2.25 & 0.14 & 0.29 \\
\hline \multicolumn{5}{|l|}{ Body Mass (Kg) } \\
\hline Group $\times$ time interaction & 0.35 & 0.94 & 0.06 & 0.15 \\
\hline Group Time Effect & 0.53 & 1.42 & 0.09 & 0.15 \\
\hline \multicolumn{5}{|l|}{ Body Fat (Kg) } \\
\hline Group $\times$ time interaction & 0.99 & 0.000 & 0.0 & 0.05 \\
\hline Group Time Effect & 0.95 & 0.005 & 0.0 & 0.05 \\
\hline \multicolumn{5}{|l|}{ Lean Body Mass (Kg) } \\
\hline Group $x$ time interaction & 0.065 & 4.01 & 0.223 & 0.46 \\
\hline Group Time Effect & $0.045^{*}$ & 4.83 & 0.256 & 0.53 \\
\hline
\end{tabular}

changes in muscle architecture (e.g. vastus lateralis thickness and pennation angle) between PA and PL were also unclear. However, it appeared very likely that PA was more beneficial for increasing lean body mass $(2.6 \%$ increase from pre to post) than PL (a $0.1 \%$ change from pre to post) (see Figure 2). Differences in the change in body mass or fat mass between PA and PL were unclear.

\section{Discussion}

This is the first study known that has examined the efficacy of phosphatidic acid on enhancing strength and muscle growth. The results of this study indicate that

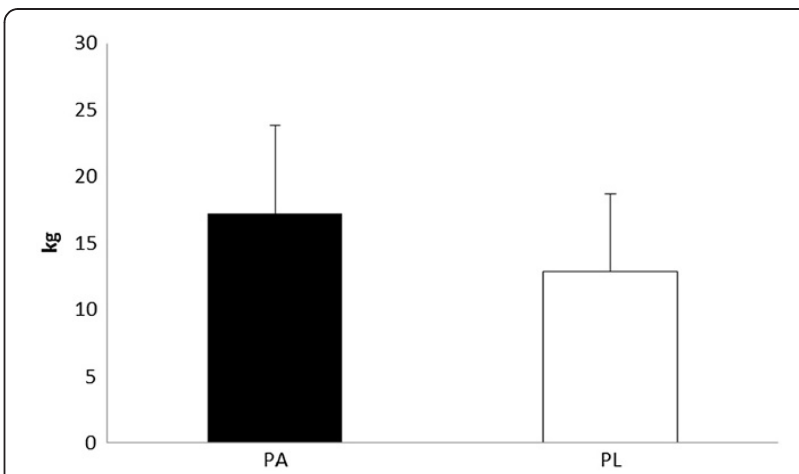

Figure 1 Changes in $\Delta \mathbf{1 - R M}$ squat strength. All data are reported as mean \pm SD.

8 weeks of supplementation with PA is likely to very likely beneficial in increasing lower body strength and lean body mass, respectively, compared to PL (Table 4). The effects of PA supplementation on upper body strength and muscle architecture were unclear. Recent evidence on rodent models have indicated that resistance exercise or an intermittent muscle stretch can activate mTORC1 by direct binding of PA to mTOR $[11,21]$. It has been suggested that the mechanical action of muscle contraction can stimulate the growth promoting pathways within muscle [22]. Considering that the mTOR signaling pathway was not examined in this study, we can only speculate on the mechanisms that may have contributed to the observed results.

The mechanical stimulus of resistance training has been demonstrated to be a potent stimulus for increasing protein synthesis $[23,24]$. If protein or essential amino acids are ingested either before or following a workout, the effect on muscle protein synthesis appears to be magnified [25]. Recent evidence has suggested that leucine, even in low dosages, may be very effective in stimulating muscle protein synthesis [26]. In consideration of the potential effects that protein ingestion has on muscle recovery and remodeling, we felt it important to provide a standardized protein supplement

Table 5 Magnitude based inferences on strength, muscle architecture and body composition changes between groups

\begin{tabular}{|c|c|c|c|c|c|}
\hline PA vs. PL & $\begin{array}{l}\text { Mean } \\
\text { difference }\end{array}$ & $\begin{array}{l}\text { Clinical } \\
\text { inference }\end{array}$ & $\begin{array}{l}\text { \% beneficial/ } \\
\text { positive }\end{array}$ & $\begin{array}{l}\% \text { negligible/ } \\
\text { trivial }\end{array}$ & $\begin{array}{l}\% \text { harmful/ } \\
\text { negative }\end{array}$ \\
\hline 1-RM Bench Press (kg) & 2.38 & Unclear & 63.5 & 0 & 36.5 \\
\hline 1-RM Squat (kg) & 4.31 & Likely & 88 & 4.8 & 7.2 \\
\hline Vastus Lateralis Thickness (cm) & .007 & Unclear & 0.25 & 99.5 & 0.25 \\
\hline Vastus Lateralis Pennation angle $\left(^{\circ}\right)$ & .79 & Unclear & 26 & 18.2 & 55.8 \\
\hline Body Mass (kg) & .006 & Unclear & 72 & 18 & 10.1 \\
\hline Body Fat (kg) & -14.5 & Unclear & 50.5 & 0 & 49.5 \\
\hline Lean Body Mass (kg) & 1.6 & Very Likely & 96.4 & 0.7 & 2.9 \\
\hline
\end{tabular}




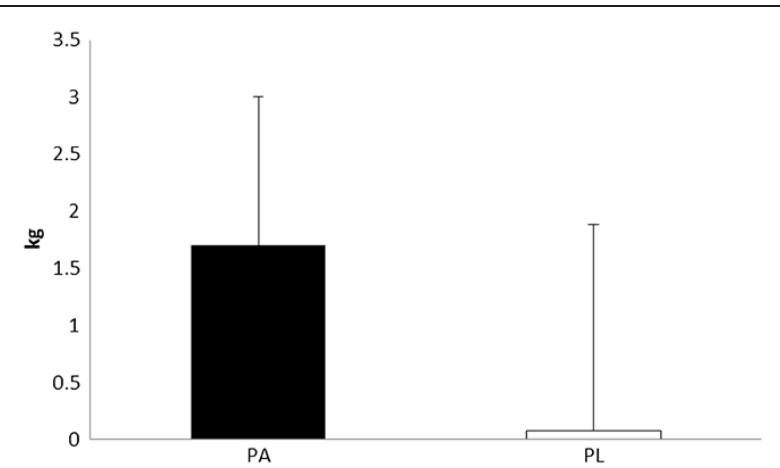

Figure $\mathbf{2}$ Changes in $\Delta$ lean body mass. All data are reported as mean \pm SD

to all subjects (both PA and PL) following each training session. With daily nutritional intake, including protein, similar between each group, the changes noted in this study (increases in lower body strength and lean body mass) likely reflect the ingestion of PA (Tables 3, 4 and 5). Since PA is thought to act on a parallel pathway to a protein stimulus (specifically leucine) on activating the mTOR pathway $[8,27]$, the greater benefit towards increases in lower body strength and lean body mass in PA suggests that the ingestion of this supplement may enhance lean tissue accruement and lower body strength to a greater extent than protein supplementation or resistance exercise only.

Differences between upper and lower body strength gains seen in this study may reflect the training experience of the subjects. Though all subjects had at least one year of resistance training experience, previous research on competitive strength power athletes has indicated that improvements in lower body strength may precede changes in upper body strength $[28,29]$. This may reflect a greater experience in upper body training and a requirement for performing the squat exercise to appropriate depth and technique. None of the subjects in the study were working with a strength coach or personal trainer prior to their enrollment into the study. Evaluation of the training logs and performance testing were conducted by certified strength and conditioning specialists that reinforced proper technique and form during the testing. Considering the skill and technique necessary for performing the squat exercise, many competitive and recreational resistance trained athletes do not perform this exercise correctly [30]. It is likely that the resistance training experience of the subjects resulted in a relative high level of performance in the bench press exercise. Although all subjects had performed the squat exercise prior to this study, their technical ability and skill for this exercise (i.e. bar placement, knee and foot alignment and lowering to parallel) varied widely. Since proper technique was stressed during the training and testing program it is possible that the subjects had a larger window of opportunity for strength gains based upon improved technique in the squat exercise compared to the bench press exercise. Thus, the strength improvements seen in the squat exercise could be partially attributed to a learning effect.

There were no clear benefits from PA ingestion in changes to muscle architecture of the vastus lateralis (Tables 3 and 5). The training program appeared to result in similar changes in muscle thickness for both groups, but did not result in any significant changes in pennation angle. The results observed in vastus lateralis thickness are similar to those reported by Blazevich and colleagues [31] following 5-weeks of training in competitive athletes, but greater than those reported by Santilla and colleagues [32] following 8-weeks of training in tactical athletes. However, the subjects in the latter study were also performing their basic military training that likely blunted maximal muscle growth. Comparisons between studies are also difficult to make due to the differences in subjects training status, the resistance training program and training duration. Although PA did appear to have a likely benefit on 1-RM squat changes, it did not have a similar effect on changes in vastus lateralis thickness. A recent study has questioned the importance of vastus lateralis changes on lower body strength performance as those investigators were unable to find any significant correlation between vastus lateralis thickness and lower body power performance [33]. The lack of any significant changes in pennation angle for either group may also be related to resistance training experience, as experience does appear to impact the magnitude of change in pennation angle [31].

There are a number of limitations associated with this study. The scientific treatise that has emanated on phosphatidic acid and its role on muscle protein synthesis stimulated the desire to examine this further. Although the results of this study provide a degree of efficacy on this novel ingredient, it does not provide any support to the previously discussed mechanisms of action. However, the results of this study do provide some evidence on the proof of concept that PA may have a role in muscle strength and lean tissue accruement. Additional research is needed to add support to these results: a bioavailability study to investigate the absorption profile of orally administered PA, a muscle biopsy study to investigate the potential increase in muscle PA content, different target groups: trained, untrained, elderly subjects, dose finding studies to investigate if the effect of PA is dose dependent, the minimum effective dose and mechanistic studies. This will have important implications for athletes participating in strength/power sports, as well as mature adults attempting to maintain muscle strength and mass as they age. 
In conclusion, the results of this study suggest that a combination of a daily $750 \mathrm{mg}$ PA ingestion, combined with a 4-day per week resistance training program for 8weeks appears to have a likely benefit on strength improvement, and a very likely benefit on lean tissue accruement in young, resistance trained individuals. Additional research is warranted to provide further elucidation on the mechanisms that govern PA and muscle protein synthesis, muscle growth and performance.

\section{Competing interests}

MP and RJ have been named as inventors on pending patents by Chemi Nutra. MP and RJ are independent paid consultants to Chemi Nutra. All other authors declare that they have no competing interests.

\section{Authors' contributions}

JRH was the primary investigator, supervised all study recruitment and data/ specimen analysis. JRH, MP and RJ designed study, JRH and JRS performed the statistical analysis, JRH supervised the manuscript preparation, JRS, DRW and RJ helped drafting the manuscript. DRW, AJW, MSF, GTM, AMG, NSE, WPM and TCS assisted with data collection and data analysis. All authors read and approved the final manuscript.

\section{Acknowledgements}

The authors would like to thank a dedicated group of subjects. This study was supported by a grant from Chemi Nutra, White Bear Lake, MN.

\section{Author details}

${ }^{1}$ Human Performance Laboratory, University of Central Florida, Orlando 32186, FL, USA. ${ }^{2}$ Increnovo LLC, 2138 E. Lafayette PI, Milwaukee 53202, WI, USA.

Received: 24 August 2012 Accepted: 26 September 2012 Published: 5 October 2012

\section{References}

1. Hanahan DJ, Nelson DR: Phospholipids as dynamic participants in biological processes. J Lipid Res 1984, 25:1528-1535.

2. Jäger R, Purpura M, Kingsley M: Phospholipids and sports nutrition. J Int Soc Sports Nutr 2007, 4:5.

3. Singer WD, Brown HA, Sternweis PC: Regulation of eukaryotic phosphatidylinositol-specific phospholipase C and phospholipase D. Annu Rev Biochem 1997, 66:475-509.

4. Lim H, Choi Y, Park W, Lee T, Ryu S, Kim S, Kim JR, Kim JH, Baek S: Phosphatidic acid regulates systemic inflammatory responses by modulationg the Akt-mamalian target of rapamycin-p70 S6 Kinase pathway. J Bio Chem 2003, 2003(278):45117-45127.

5. Andresen BT, Rizzo MA, Shome K, Romero G: The role of phosphatidic acid in the regulation of the Ras/MEK/Erk signaling cascade. FEBS Lett 2002, 531:65-68.

6. McDermott M, Wakelam JM, Morris AJ: Phospholipase D. Biochem Cell Biol 2004, 82:225-253.

7. Xu Y, Fang Y, Chen J, Prestwich G: Activation of mTOR signaling by novel fluoromethylene phosphonate analogues of phosphatidic acid. Bioorg Med Chem Lett 2004, 14:1461-1464.

8. Fang Y, Vilella-Bach M, Bachmann R, Flanigan A, Chen J: Phosphatidic acidmediated mitogenic activation of mTOR signaling. Science 2001, 294:1942-1945.

9. Xiaochun B, Jiang Y: Key factors in mTOR regulation. Cell Mol Life Sci 2009, 67:239-253.

10. Koopman R: Role of amino acids and peptides in the molecular signaling in skeletal muscle after resistance exercise. Int J Sport Nutr Exerc Metab 2007, 17(Suppl):S47-S57.

11. Hornberger T, Chu W, Mak Y, Hsiung J, Huang S, Chien S: The role of phospholipase $\mathrm{d}$ and phoshatidic acid in the mechanical activation of mTOR signaling in skeletal muscle. Proc Natl Acad Sci 2006, 103:4741-4746

12. Lehman N, Ledford B, Di Fulvio M, Frondorf K, McPhail L, Gomez-Cambroner G: Phospholipase D2-derived phosphatidic acid binds to and activates ribosomal p70 S6 Kinase independently of mTOR. FASEB J 2007, 21:1075-1094

13. Hoffman JR: Norms for Fitness, Performance, and Health. Champaign: Human Kinetics; 2006.

14. Hoffman JR, Fry AC, Deschenes M, Kraemer WJ: The effects of self-selection for frequency of training in a winter conditioning program for football. J Appl Sport Sci Res 1990, 4:76-82.

15. Hoffman JR, Fry AC, Howard R, Maresh CM, Kraemer WJ: Strength, speed, and endurance changes during the course of a division I basketball season. J App/ Sport Sci Res 1991, 5:144-149.

16. Klimstra M, Dowling J, Durkin JL, MacDonald M: The effect of ultrasound probe orientation on muscle architecture measurement. J Electromyogr Kinesiol 2007, 17:504-514.

17. Abe T, Fukashiro S, Harada Y, Kawamoto K: Relationship between sprint performance and muscle fascicle length in female sprinters. J Physio Anthropol Appl Human Sci 2001, 20:141-147.

18. Green SB, Salkind NJ, Akey TM: Using SPSS for Windows: Analyzing and Understanding Data. 2nd edition. Upper Saddle River: Prentice Hall; 2000

19. Batterham AM, Hopkins WG: Making meaningful inferences about magnitudes. Int J Sports Physiol Perf 2006, 1:50-57.

20. Hopkins WG, Batterham AM, Marshall SW, Hanin J: Progressive statistics. Sportscience 2009, 13:55-70.

21. O' Neil TK, Duffy LR, Frey JW, Hornberger TA: The role of phosphoinositide 3kinas and phosphatidic acid in the regulation of mammalian target of rapamycin following eccentric contractions. J Physiol 2009, 587:3691-3701.

22. Rasmussen B: Phosphatidic acid: a novel mechanical mechanism for how resistance exercise activates mTORC1 signaling. J Physiol 2009, 587:3415-4316.

23. Biolo G, Maggi SP, Williams BD, Tipton KD, Wolfe RR: Increased rates of muscle protein turnover and amino acid transport after resistance exercise in humans. Am J Physiol Endocrinol 1995, 268:E514-E520.

24. Roy BD, Fowles JR, Hill R, Tarnopolsky MA: Macronutrient intake and whole body protein metabolism following resistance exercise. Med Sci Sports Exerc 2000, 32:1412-1418.

25. Tipton KD, Wolfe RR: Exercise, protein metabolism, and muscle growth. Int J Sport Nutr Exerc Metab 2001, 11:109-132.

26. Churchward-Venne TA, Burd NA, Mitchell CJ, West DWD, Philp A, Marcotte GR, Baker SK, Baar K, Phillips SM: Supplementation of a suboptimal protein dose with leucine or essential amino acids: effects on myofibrillar protein synthesis at rest and following resistance exercise in men. J Physiol 2012, 590:2751-2765.

27. Winter JN, Fox TE, Kester M, Jefferson LS, Kimball SR: Phosphatidic acid mediates activation of mTORC1 through the ERK signaling pathway. Am J Physiol Cell Physiol 2010, 299:C335-C344.

28. Hoffman JR, Kang J: Strength changes during an inseason resistance training program for football. J Strength Cond Res 2003, 17:109-114.

29. Hoffman JR, Wendell M, Cooper J, Kang J: Comparison between linear and nonlinear inseason training programs in freshman football players. J Strength Cond Res 2003, 17:561-565.

30. Miletello WM, Beam JR, Cooper ZC: A biomechanical analysis of the squat between competitive collegiate, competitive high school, and novice powerlifters. J Strength Cond Res 2009, 23:1611-1617.

31. Blazevich AJ, Gill ND, Bronks R, Newton RU: Training-specific muscle architecture adaptation after 5-wk training in athletes. Med Sci Sports Exerc 2003, 35:2013-2022.

32. Santtila $M$, Kyrolainen $H$, Hakkinen $K$ : Changes in maximal and explosive strength, electromyography, and muscle thickness of lower and upper extremities induced by combined strength and endurance training in soldiers. J Strength Cond Res 2009, 23:1300-1308.

33. Earp JE, Joseph M, Kraemer WJ, Newton RU, Comstock BA, Fragala MS, Dunn-Lewis C, Solomon-Hill G, Penwell ZR, Powell MD, Volek JS, Denegar CR, Häkkinen K, Maresh CM: Lower-body muscle structure and its role in jump performance during squat, countermovement, and depth drop jumps. J Strength Cond Res 2010, 24:722-729.

doi:10.1186/1550-2783-9-47

Cite this article as: Hoffman et al.: Efficacy of phosphatidic acid ingestion on lean body mass, muscle thickness and strength gains in resistance-trained men. Journal of the International Society of Sports Nutrition 2012 9:47 